\title{
EndoG is dispensable in embryogenesis and apoptosis
}

\author{
KK David ${ }^{1,2,3}$, M Sasaki ${ }^{1,2}$, S-W Y ${ }^{1,2}$, TM Dawson $^{1,2,3,4}$ and \\ VL Dawson*,1,2,3,4,5 \\ 1 Institute for Cell Engineering Program in Neuroregeneration and Repair, Johns \\ Hopkins School of Medicine, Baltimore, MD 21205, USA \\ 2 Department of Neurology, Johns Hopkins School of Medicine, Baltimore, MD \\ 21205, USA \\ ${ }^{3}$ Graduate Program in Cellular and Molecular Medicine, Johns Hopkins School \\ of Medicine, Baltimore, MD 21205, USA \\ 4 Department of Neuroscience, Johns Hopkins School of Medicine, Baltimore, \\ MD 21205, USA \\ 5 Department of Physiology, Johns Hopkins School of Medicine, Baltimore, MD \\ 21205, USA \\ * Corresponding author: VL Dawson, Institute for Cell Engineering, Johns \\ Hopkins University School of Medicine, 733 North Broadway, Suite 731, \\ Baltimore, MD 21205, USA. Tel: + 1410614 3361; fax: + 1410614 9568; \\ E-mail: vdawson@jhmi.edu
}

Received 18.3.05; revised 02.8.05; accepted 06.9.05; published online 21.10.05 Edited by P Vandenabeele

\begin{abstract}
The mitochondrial protein, endonuclease $G$ (EndoG), is one of the endonucleases implicated in DNA fragmentation during apoptosis. It has been shown to translocate from the mitochondria to the nucleus upon cell death stimuli. These observations suggest that EndoG is a mitochondrial cell death effector, and that it possibly acts as a cell death nuclease, similar to DNA fragmentation factor. To better understand the role of EndoG in development and apoptosis, we generated EndoG null mice by homologous gene targeting without disruption of D2Wsu81e. EndoG null mice are viable and develop to adulthood with no obvious abnormalities. Fibroblasts generated from the EndoG null mice show no difference in susceptibility when induced to die by a variety of intrinsic and extrinsic apoptotic stimuli. Additionally, EndoG null mice are equally sensitive to excitotoxic stress. These data suggest that EndoG is not essential for early embryogenesis and apoptosis.

Cell Death and Differentiation (2006) 13, 1147-1155.

doi:10.1038/sj.cdd.4401787; published online 21 October 2005
\end{abstract}

Keywords: endonuclease; apoptosis; DNA fragmentation

Abbreviations: EndoG, endonuclease G; DFF, DNA fragmentation factor; AIF, apoptosis-inducing factor; NMDA, $N$-methyl-D-aspartate; MNNG, $N$-methyl- $N$-nitro- $N$-nitrosoguanidine; cisplatin, cis-platinum(II) diamminedichloride; TNF- $\alpha$, tumor-necrosis factor-alpha; DAPI, 4', 6-diamidino-2-phenylindole; $\mathrm{CHX}$, cycloheximide; PI, propidium iodide

\section{Introduction}

Apoptosis is a physiologic cell death program that is essential for normal embryonic development, maintenance of tissue homeostasis, and appropriate physiologic response to tissue injury. Dysregulation of apoptosis can result in abnormal embryonic development and can contribute to various disease conditions including neurodegeneration, autoimmunity and cancer. A large body of work has accumulated regarding the biochemical steps required for apoptosis. Apoptosis can occur through activation of cysteine proteases, caspases, in a protease cascade of initiators and effectors that trigger the morphological changes associated with the apoptotic phenotype. Through cleavage of DNA fragmentation factor 45 (DFF45), caspases facilitate the translocation of DNA fragmentation factor (DFF) into the nucleus resulting in the classic DNA fragmentation pattern of oligonucleosomal DNA fragments that is the biochemical hallmark of apoptosis. ${ }^{1-4}$ Therefore, cleavage of DFF45 by caspases links caspase activation to DNA fragmentation. However, genetically engineered mice lacking DFF45 or site-directed mutants of DFF45 lacking the caspase-cleavage site, still retain residual DNA fragmentation and are phenotypically normal. ${ }^{5,6}$ These observations led to the identification and characterization of endonuclease $\mathrm{G}$ (EndoG). Studies have shown that EndoG is activated during cytotoxic death to induce chromosomal DNA fragmentation. EndoG is localized in the mitochondria, and then translocates to the nucleus during apoptosis with cell death stimuli such as truncated Bid (tBid), tumor-necrosis factor-alpha (TNF- $\alpha$ ) and UV irradiation. ${ }^{6,7}$ Thus, EndoG joins other mitochondrial proteins including cytochrome $c$, Omi/ HtrA2, Smac/DIABLO and apoptosis-inducing factor (AIF) as cell death effectors that are sequestered in the mitochondria, but released during the cell death process. However, the exact role and relevance of EndoG in apoptosis is controversial.

EndoG is a mitochondrial nuclease that was first identified and characterized in yeast as NUC1. ${ }^{8-10}$ EndoG, a wellconserved nuclease, is a homodimer that is thought to be involved in mitochondrial DNA replication ${ }^{11}$ with important roles in recombination and repair. ${ }^{12,13}$ Mitochondrial endonuclease activity varies among tissues and appears to be related to the rate of tissue metabolism. ${ }^{14}$ While EndoG is believed to be localized in the mitochondria, its exact function and location within mitochondria are not clear. In yeast, the NUC1 knockout cells have normal mitochondrial function. ${ }^{15}$ In Caenorhabditis elegans, genetic knockout of cps- 6 or RNAmediated interference of cps- 6 affects normal DNA degradation and results in the delayed appearance of cell corpses during development. ${ }^{16}$ To investigate EndoG function in mammalian systems, we generate and characterize EndoG null mice. The EndoG null mice are viable and develop to adulthood with no obvious abnormalities. This is in contrast with a previous report describing embryonic lethality in EndoG null mice, ${ }^{17}$ but agrees with a recent report describing viability and lack of obvious abnormalities in EndoG null mice. ${ }^{18} \mathrm{We}$ extend the latter study by treating mouse embryonic fibroblasts with different cell death stimuli and by subjecting EndoG null mice to excitotoxicity. Fibroblasts generated from 
the EndoG null mice undergo normal apoptosis to a variety of apoptotic stimuli. Additionally, EndoG null mice are equally sensitive to excitotoxic stress.

\section{Results}

\section{Generation of EndoG null mice}

The EndoG targeting vector was constructed with a mouse phosphoglycerate kinase 1 (PGK) neomycin (NEO) expression cassette spanning exon 2 and part of exon 3 (Figure 1a). ES cells were electroporated with linearized PGK-NEO and subjected to $\mathrm{G} 418$ selection. Resistant clones were screened for the targeted EndoG locus and these clones were subsequently injected into blastocysts. Chimeric mice were generated and bred with ICR mice resulting in successful germ line transmission yielding EndoG heterozygote mice. Heterozygote mating resulted in litters with expected Mendelian ratios of EndoG genotypes including EndoG null mice. Southern Blot analysis (Figure 1b) demonstrates the expected disrupted allele in the EndoG null mice and both alleles in the heterozygote EndoG mice. Northern Blot

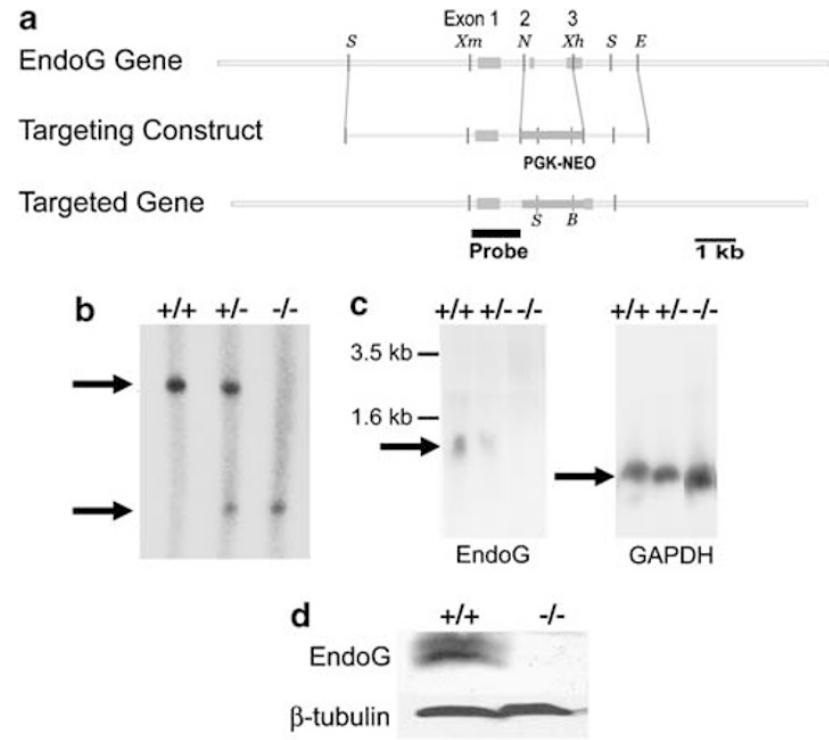

Figure 1 Generation of EndoG null mice. (a) The genomic organization of the EndoG gene, the EndoG targeting vector, and the expected disrupted allele after homologous recombination. The targeting vector is comprised of the PGK-neobpA cassette (NEO), a 5'-flanking long arm of 6-kb Spel-Nhel fragment, a $3^{\prime}$ flanking short arm of the1.8-kb Xhol-EcoRl fragment and a herpes simplex virus thymidine kinase (hsv-tk) gene for negative selection. Homologous recombination of the targeting vector introduces the NEO cassette deleting the $2.0-\mathrm{kb}$ Spel$E c o R I$ region containing exons 2 and part of exon 3 , which encode the $c$-terminal region of EndoG containing the active site. The restriction sites for Spel (S), Xmnl $(\mathrm{Xm}), \mathrm{Xhol}(\mathrm{Xh})$, Nhel $(\mathrm{N}), \mathrm{EcoRI}(\mathrm{E}), \mathrm{BamHI}(\mathrm{B})$ along with the probe used for Southern blotting are indicated. (b) Southern blot analysis of genomic DNA from wild-type and EndoG null mice. The probe indicated in (a) was hybridized to genomic DNA digested by $X \mathrm{mnl}$ and Spel. A $1.5-\mathrm{kb}$ restriction fragment is diagnostic for the targeted allele in contrast to the $3.5-\mathrm{kb}$ fragment generated from the wild-type allele. (c) Northern Analysis of EndoG expression using total RNA extracted from the brain of wild-type, heterozygote and EndoG null mice. These data were repeated at least three times with similar results. (d) Western analysis of EndoG expression by using extracts from brain with $\beta$-tubulin as loading control. These data were repeated at least three times with similar results analysis reveals loss of the EndoG mRNA in the EndoG null mice (Figure 1c). Western Blot analysis confirms the loss of EndoG protein in the EndoG null mice (Figure 1d).

\section{D2Wsu81e remains intact in the EndoG null mice}

Zhang et al. $^{17}$ reported EndoG null mice to be embryonic lethal, whereas the EndoG knockout described here results in viable mice. The $3^{\prime}$ end of exon 3 of the EndoG gene overlaps with the last exon of D2Wsu81e (locus identification number 227695). The function of D2Wsu81e is unknown. The first report of EndoG null mice described an embryonic lethal phenotype, but the mice also had a substantial part of the D2Wsu81e gene knocked out. The EndoG null mice described here results in viable mice. Taken together, these observations suggest that D2Wsu81e is essential for embryogenesis and embryonic viability. Our targeting strategy and the genetic localization of D2Wsu81e in comparison to EndoG are represented in Figure $2 a$ indicating only part of the $3^{\prime}$ untranslated region of D2Wsu81e was disrupted. To confirm that expression of the coding region of D2Wsu81e was not disrupted in the EndoG null mice, RT-PCR to detect the D2Wsu81e mRNA was conducted (Figure 2b). There is no significant difference in the expression of mRNA for D2Wsu81e between wild-type and EndoG null mice, whereas the mRNA for EndoG in EndoG null mice is absent by RT-PCR.

\section{Submitochondrial localization of EndoG}

The mitochondrial localization of EndoG has been postulated to be in the intermembrane space, but studies have been equivocal. Some studies suggested that EndoG is localized in the intermembrane space, ${ }^{19}$ thus favoring efficient release during the apoptotic process. ${ }^{6}$ This is in contrast with a study observing a limited mitochondrial release of EndoG consistent with the nuclease tethered to the inner membrane or localized

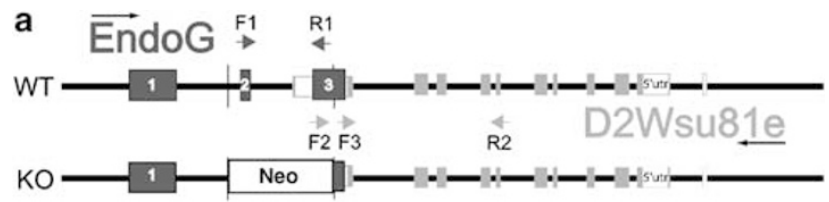

b

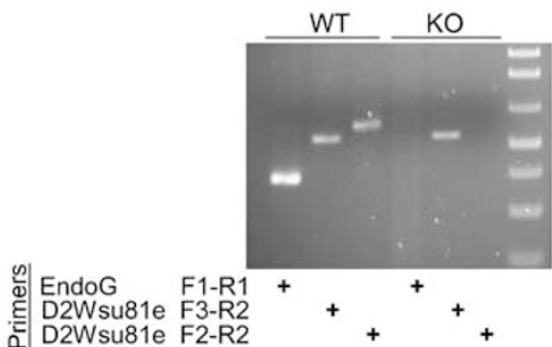

Figure 2 Overlap of EndoG and D2Wsu81e. (a) Schematic representation of the gene structure of EndoG and D2Wsu81e. Primers used for RT-PCR are indicated: EndoG sense primer (F1), antisense primer (R1), D2Wsu81e sense primer (F2) antisense 1 (R2), antisense 2 (R3). (b) Confirmation of D2Wsu81e expression by RT-PCR in wild-type, heterozygote and EndoG null mice. Results are representative of at least three experiments 
in the mitochondrial matrix..$^{20,21}$ To definitively localize EndoG in the mitochondria, we conducted fractionation of mouse brain mitochondria using standard markers for each of the submitochondrial fractions including VDAC for outer membrane, cytochrome $c$ for intermembrane space, COX IV for inner membrane, Hsp60 for matrix (Figure 3a). We find that EndoG is localized primarily in the inner membrane and matrix. In addition, EndoG, but not COX IV, is released from the inner membrane upon treatment with $\mathrm{Na}_{2} \mathrm{CO}_{3}$ suggesting that EndoG is tethered to the inner membrane (Figure $3 b$ ).

\section{Morphologic characterization of organs and tissues of EndoG null mice}

There is no discernable physical difference between EndoG null mice and wild-type littermate controls. On gross anatomic dissection there are also no obvious abnormalities between wild-type littermates and EndoG null mice at two months of age. A detailed histologic analysis of various organs was conducted to determine if there were cellular differences between wild-type and EndoG null mice. Sections were taken from spleen, thymus, heart, liver, testes and stomach (Figure 4). Additionally, kidney, lung, pancreas, Peyer's patches and various regions of the Gl tract were also evaluated (data not shown). Upon microscopic analysis all organ tissues are unremarkable and there are no differences between wild-type and EndoG null mice.

Developmental apoptosis or programmed cell death is critical for the normal development of the brain. Therefore, detailed anatomic sections of the brain were obtained from

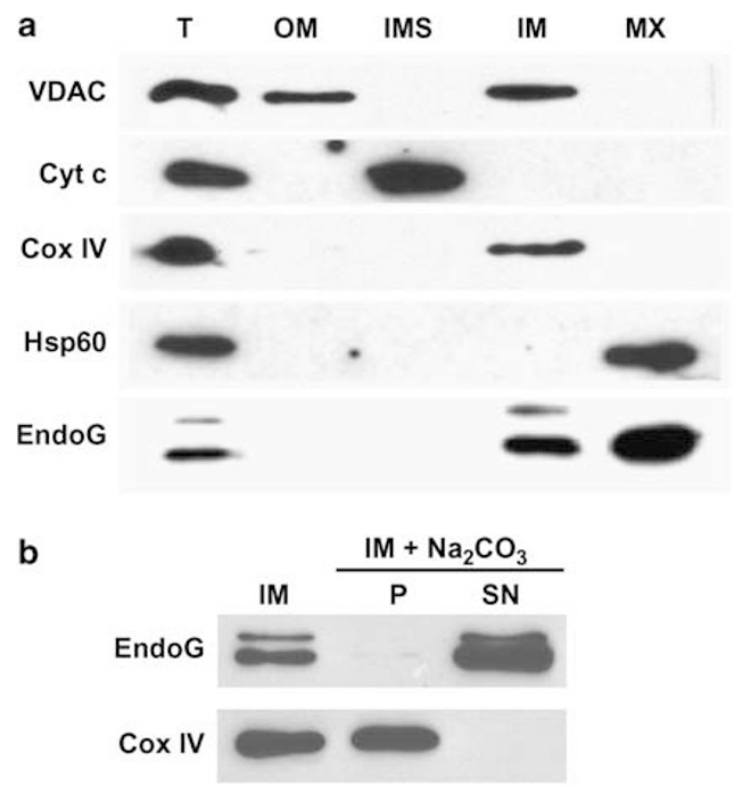

Figure 3 Mitochondrial Localization of EndoG. (a) EndoG is localized in the inner membrane and the matrix. Submitochondrial localization of EndoG was determined using standard markers for mitochondrial compartments: VDAC for outer membrane, cytochrome $c$ for intermembrane space, COX IV for inner membrane, Hsp60 for matrix. T-total, OM-outer membrane, IMS-intermembrane space, IM-inner membrane, MX-matrix. (b) EndoG is tethered to the inner membrane and can be released to the soluble fraction with $\mathrm{Na}_{2} \mathrm{CO}_{3}$. IM-inner membrane, P-pellet/insoluble fraction, SN-supernatant/soluble fraction rostral to caudal in 2-month-old mice. There are no differences between wild-type and EndoG null mice in the neuroanatomy of the brain (Figure 5).

\section{Apoptosis is normal in primary mouse embryonic fiboblasts from wild type and EndoG null mice}

Apoptotic stimuli such as TNF- $\alpha$, tBID and UV irradiation results in the release of EndoG from the mitochondria, and this release would allow EndoG to participate in chromosomal

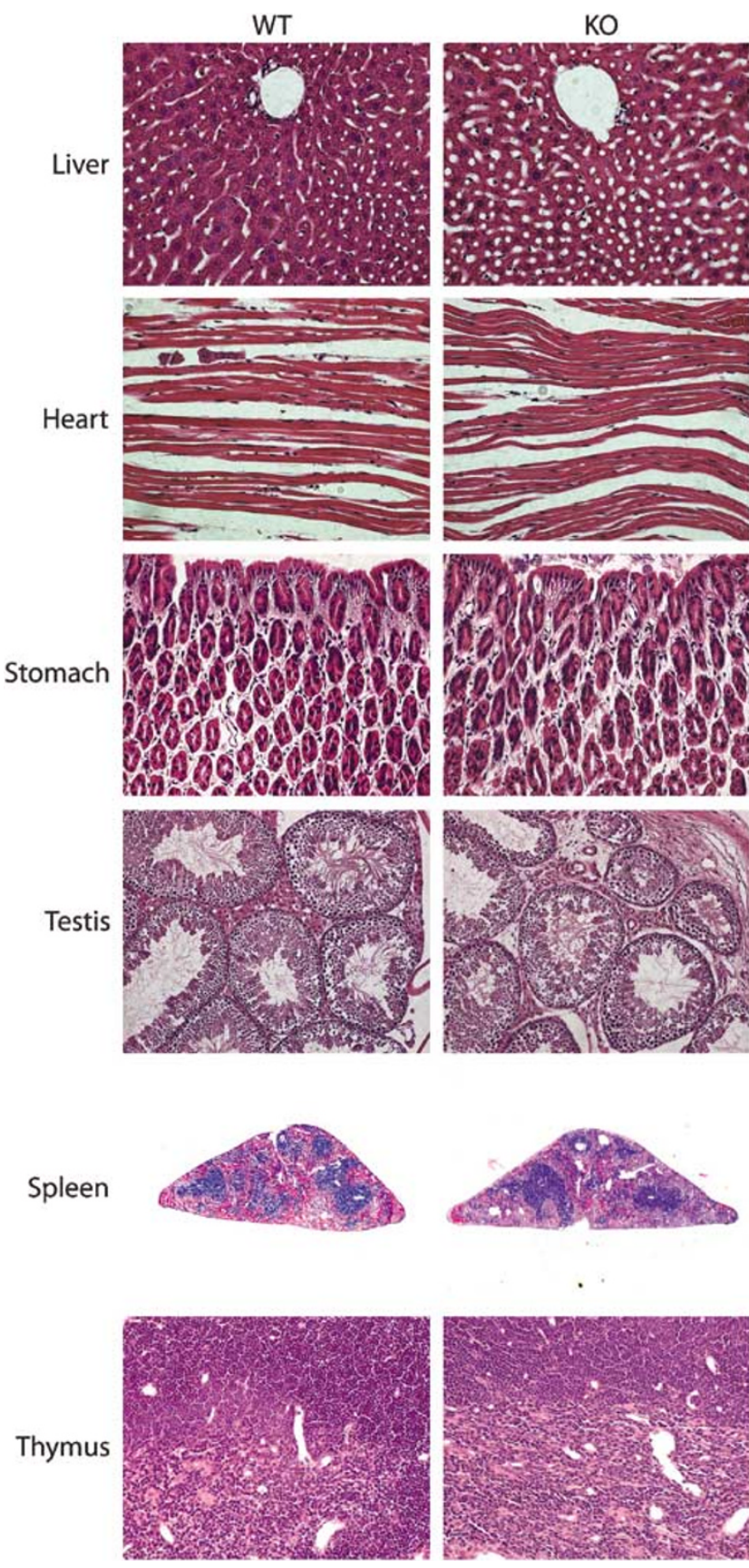

Figure 4 Representative sections of spleen, thymus, heart, liver, testes and stomach from wild-type and EndoG null mice are shown following hematoxylineosin staining $(n=3)$ 
a

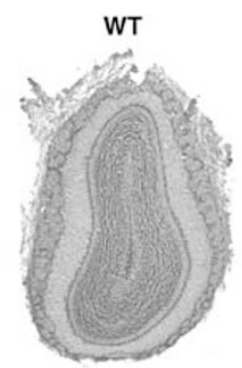

C

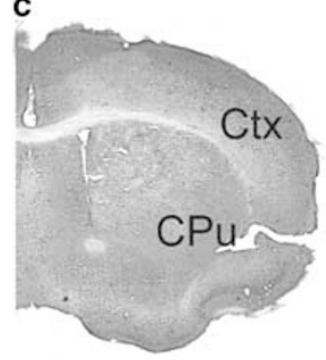

e

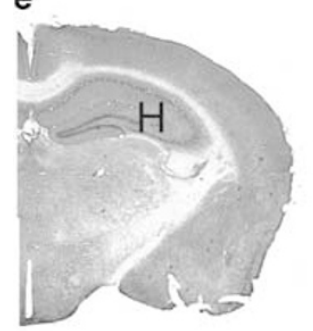

g

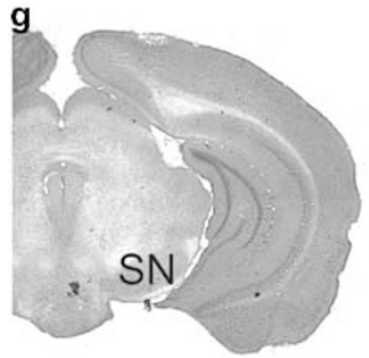

i

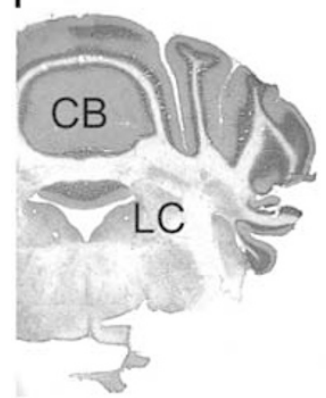

b

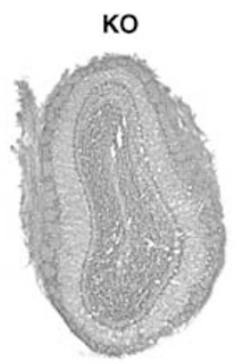

d

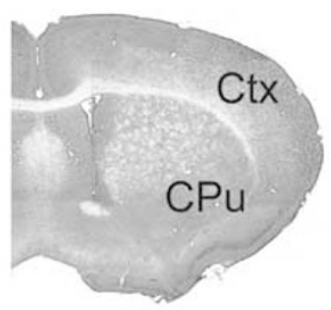

f

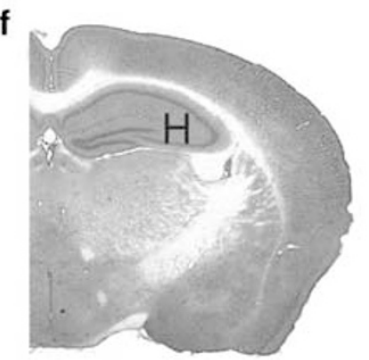

h

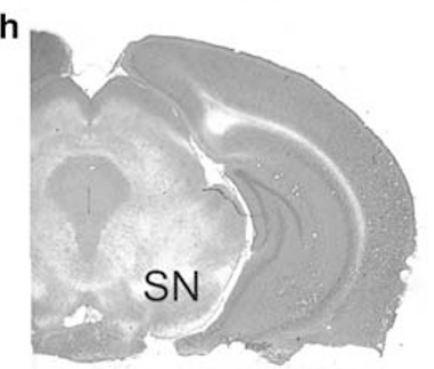

j

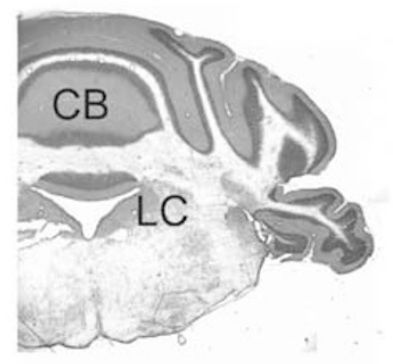

Figure 5 Representative coronal sections of the brain from wild-type and EndoG null mice are shown following hematoxylin-eosin staining $(n=3):(\mathbf{a}, \mathbf{b})$ Olfactory bulb; (c, d) Sections showing the caudate putamen (CPu) and Cortex (Ctx); (e, f) Hippocampus $(\mathrm{H}) ;(\mathbf{g}, \mathbf{h})$ Sections through the midbrain showing the substantia nigra (SN); and (i, j) locus coeruleus (LC) and Cerebellum (Cb)

DNA fragmentation in normal apoptosis. ${ }^{6,7,22}$ To explore the potential role of EndoG in apoptosis, a range of chemical agents which trigger cell death in different manners were

tested in wild-type and EndoG null mouse embryonic fibroblasts (MEFs), and cell death was determined by propidium iodide staining (Figure 6a). $\mathrm{N}$-methyl- $\mathrm{N}$-nitro- $\mathrm{N}$ nitrosoguanidine (MNNG) is a DNA-damaging agent. ${ }^{23}$ Cytotoxicity by cis-platinum(II) diamminedichloride (cisplatin) is due to its interaction with DNA to form DNA adducts. ${ }^{24}$ Etoposide induces DNA breaks by inhibiting the ability of topoisomerase II to ligate cleaved nucleic acid molecules. ${ }^{25}$ Menadione is a quinone that generates quinone radicals and free radicals in mitochondria resulting in reactive oxygen species stress. ${ }^{26}$ Thapsigargin and tunicamycin induce endoplasmic reticulum-stress. ${ }^{27}$ Staurosporine (STS) is a protein kinase $\mathrm{C}$ inhibitor. ${ }^{28} \mathrm{TNF}-\alpha$ triggers apoptosis through activation of the TNF- $\alpha$ receptor and direct activation of the caspase cascade. ${ }^{29}$ Wild-type and EndoG null MEFs showed equal sensitivity to these intrinsic and extrinsic activators of apoptosis. In addition, wild-type and EndoG null MEFS displayed comparable amount of AIF that translocated to the nucleus upon treatment with MNNG (Figure 7), a potent inducer of AIF release from the mitochondria. ${ }^{23}$

Excitotoxicity mediated via the $\mathrm{N}$-methyl-D-aspartate (NMDA) receptor in the brain is thought to mediate neurotoxicity in a variety of neurologic diseases, stroke and trauma. ${ }^{23}$ Excitotoxic concentrations of NMDA were directly injected into the striata of wild-type and EndoG null mice (Figure 6b). There is no difference in lesion volume between wild type and EndoG null mice.

\section{Discussion}

The major findings of this study are that genetic disruption of the EndoG gene has no effect on normal embryogenesis or development, and that loss of EndoG function does not alter apoptosis induced by a variety of intrinsic and extrinsic apoptotic stimuli. In this study, we have deleted exon 2 and part of exon 3 of the EndoG gene. This targeting strategy is important due to the overlap of the exon 3 of the EndoG gene with part of the last exon of D2Wsu81e. The gene D2Wsu81e is expressed at high levels in extraembryonic tissues of 7.5d.p.c. embryos ${ }^{30}$ and during the four-cell embryo stage. ${ }^{31}$ Our results differ from a report of an EndoG knockout describing embryonic lethality ${ }^{17}$ in which they targeted all of the exons of EndoG including a substantial portion of D2Wsu81e (five of the 10 exons were deleted). Thus, the original description of early embryonic lethality in EndoG knockout mice is due to the inadvertent double gene disruption of EndoG and D2Wsu81e. Our study in conjunction with the recent description of another EndoG null line with no obvious abnormalities ${ }^{18}$ substantiates that EndoG is dispensable for normal embryogenesis and normal development.

A surprising finding of this current study is the lack of alterations in the response of EndoG null cells to apoptotic stimuli. EndoG is hypothesized to be a mitochondrial cell death effector that acts as an apoptotic endonuclease when released from the mitochondria upon cell death stimuli. ${ }^{6,7}$ Biochemical studies have confirmed this release $e^{6,7,20,21,32}$ with some observations suggesting that release requires caspase activation ${ }^{21}$ whereas others observed a limited release that suggests a localization in the inner membrane 
a
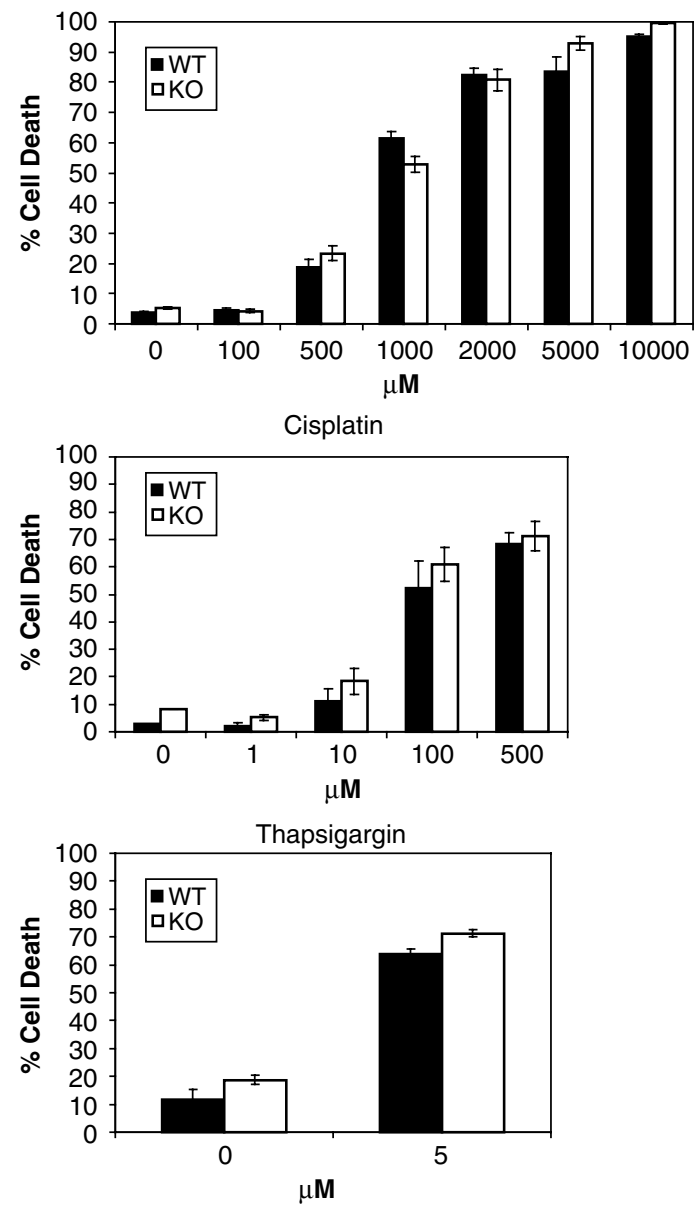

STS

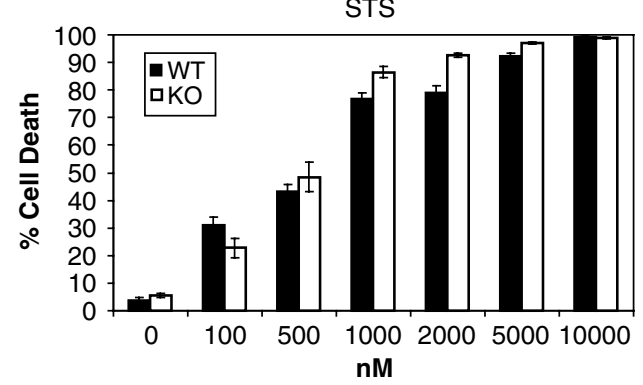

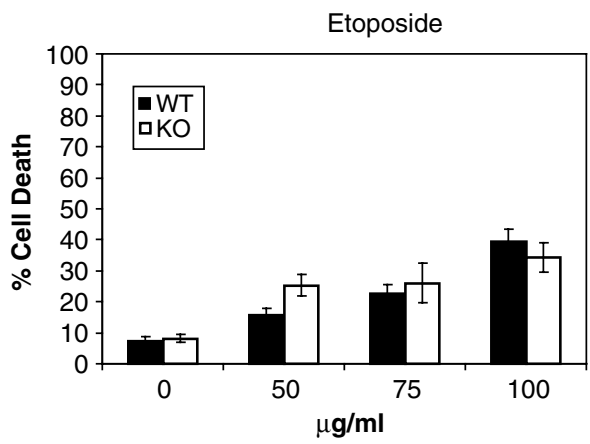
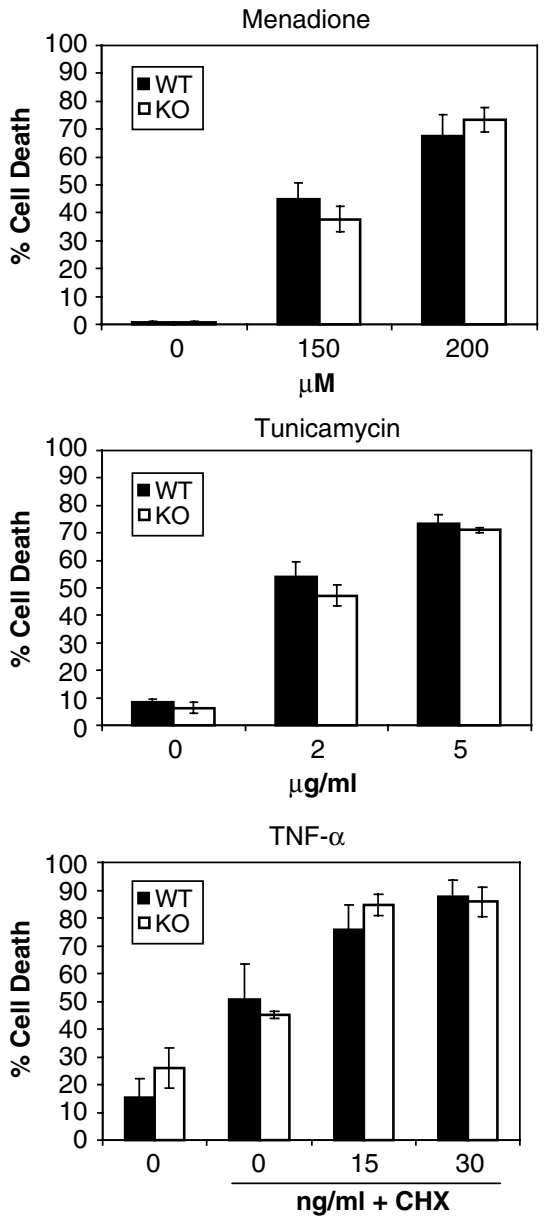

b

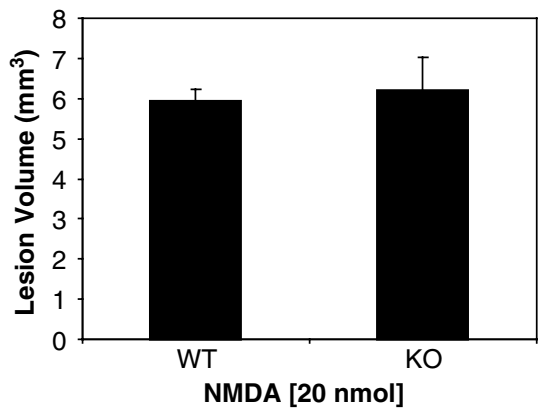

Figure 6 Cell death response in EndoG null MEFs and EndoG null mice. (a) MEFs were treated with MNNG, etoposide, cisplatin, menadione, thapsigargin, tunicamycin, staurosporine and TNF- $\alpha$ with $\mathrm{CHX}$. Cell death was assayed by PI staining. Data shown is representative of at least three separate experiments. Lack of significance was determined by Student's t-test. (b) Average lesion volumes from wild-type $(n=3)$ and EndoG null $(n=3)$ mice $48 \mathrm{~h}$ after injection of $20 \mathrm{nmol}$ NMDA. Lack of significance was determined by Student's $t$-test 

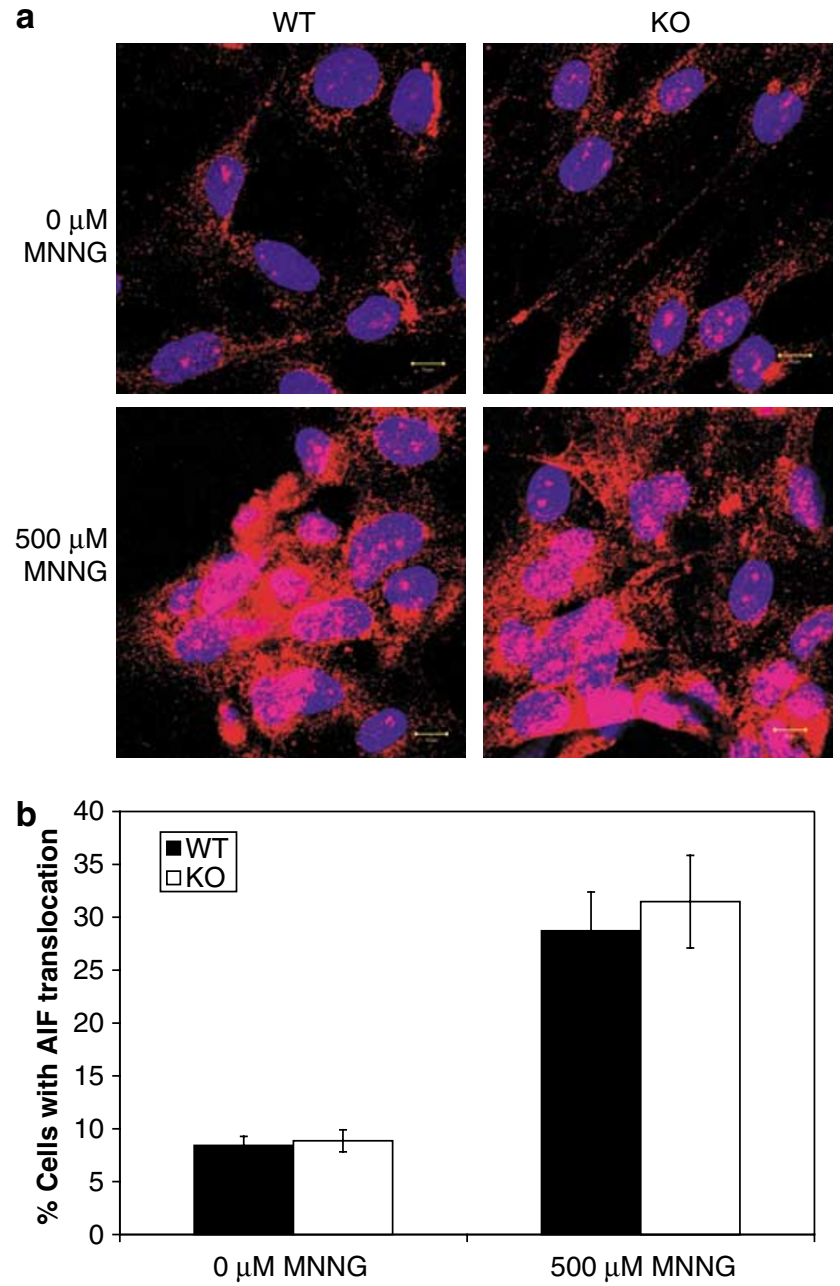

Figure 7 AIF translocation in wild-type and EndoG null MEFs. (a) MEFs treated with $500 \mu \mathrm{M}$ MNNG showed AlF translocation to the nucleus when immunostained for AIF and counterstained with DAPI. (b) The number of cells undergoing AIF translocation to the nucleus is not significantly different between wild-type and EndoG null MEFs. Data shown is representative of at three independent experiments. Lack of significance was determined by Student's $t$-test

or the mitochondrial matrix. ${ }^{20,21}$ Our submitochondrial fractionation results showed that EndoG is localized primarily in the mitochondrial matrix and is tethered to the inner membrane. We did not observe an inner membrane space localization for EndoG, which would facilitate a role for EndoG in nuclear apoptosis, but instead, we observed a matrix localization which is consistent with the function of EndoG to regulate mitochondrial DNA. These results are not consistent with the previous observation that EndoG is located in the inner membrane space. ${ }^{19}$ This discrepancy in findings may be due to a difference in the tissue source of mitochondria or the technique in preparing mitochondrial fractions. Our results, however, are consistent with the observation in this study that EndoG is dispensable for cell death. Furthermore, these results may explain the lack of detectable difference in chromosomal DNA fragmentation between wild-type and EndoG null cells upon apoptotic induction, ${ }^{18}$ and similarly, the difference in the kinetics of mitochondrial release of
EndoG compared with cytochrome $c$ upon apoptotic induction by proapoptotic $\mathrm{Bcl}-2$ proteins. ${ }^{21}$

In C. elegans, knockdown of cps-6 affected DNA fragmentation and appearance of cell corpses during development. ${ }^{16,33}$ Studies further showed that CPS-6 interact cooperatively with the $C$. elegans apoptosis-inducing factor (AIF) homolog, WAH-1, to promote DNA degradation, and that this interaction is partially dependent on the caspase-3 homolog CED-3. ${ }^{34}$ These studies suggest that EndoG may have both caspase-dependent and caspase-independent actions in cell death. However, in mammalian cells, apoptosis was unaffected between wild-type and EndoG null cells exposed to a variety of intrinsic and extrinsic apoptotic stressors including genotoxic agents such as MNNG, etoposide and cisplatin; free radical generator, menadione; ER stressors such as thapsigargin and tunicamycin; protein kinase $\mathrm{C}$ inhibitor, staurosporine, and extrinsic apoptotic activator, TNF- $\alpha$. Additionally, there was no difference in lesion volume following excitotoxicity between wild-type and EndoG null mice. Our results suggest that EndoG is not necessary for the execution of apoptosis. It is possible that other molecules such as AIF, DFF, DNase I or DNase II are able to compensate for the loss of EndoG function. However, the percentage of cells undergoing AIF translocation to the nucleus with MNNG treatment is the same for wild-type and EndoG null cells as shown by immunohistochemistry.

Since the DFF45 null mice also lack a phenotype in development and sensitivity to apoptosis, ${ }^{5}$ it is highly possible that these endonucleases compensate for each other. In order to understand the role of these endonucleases, it may be necessary to generate double mutant mice lacking both EndoG and DFF to clarify the precise physiologic role for the endonuclease in apoptosis. Additionally in development, it is hypothesized that phagocytosis is a critical component in which the DNAse II plays a critical role. ${ }^{35}$ Therefore, the existence of multiple redundant pathways may obscure understanding the role of a single endonuclease through targeted disruption.

In conclusion, despite an intriguing potential role for EndoG in mediating apoptosis, the EndoG null mice do not have any obvious defects in development or in the regulation of apoptosis.

\section{Materials and Methods}

\section{Construction of targeting vector}

Using a $300 \mathrm{bp}$ fragment from the exon 1 of murine EndoG cDNA as a probe, a genomic clone was isolated from a 129SvJ bacterial artificial chromosome (BAC) library (Invitrogen). A 9.0-kb Spel subclone spanning the entire EndoG genome was cloned from the genomic BAC clone into the Spel site of the pBluescript SK (Stratagene) vector. The cloning site of pBluescript was modified by the introduction of an Rsrll-Nhel-Ascl polylinker into its EcoRI site. An Rsrll-Rsrll fragment containing the herpes simplex virus tk gene (HSV-tk) and a Ascl-Ascl fragment containing the PGK-Neo-bGHpA cassette were subcloned from pKOselectTK and pKO-selectNeo (Stratagene) to the Rsill and Ascl sites, respectively, and designated pTK-Neo. To generate an EndoG targeting vector, an Spel-Nhel fragment of $6.5 \mathrm{~kb}$ and a Xhol-Xhol fragment of 
$1.2 \mathrm{~kb}$ was subcloned from the pEndoG-Spel-9.0 kb into the Nhel and Xhol site of the pTK-Neo, respectively (see Figure 1a).

\section{Generation of EndoG null mice}

The targeting vector was linearized with Notl, and $30 \mu \mathrm{g}$ of DNA was electroporated into embryonic stem (ES) cells derived from a mouse line of 129/SvEvTec (MC1, Johns Hopkins University Transgenic Core Laboratory) with a Bio-Rad Gene pulsar (320 V, $250 \mu \mathrm{F})$. After electroporation, $5 \times 10^{7}$ cells were plated in 10 of $100-\mathrm{mm}$-diameter tissue culture dishes containing a monolayer of G418 resistance embryonic fibroblast feeder cells and selected in Geneticin (200 $\mu \mathrm{g} / \mathrm{ml}$ active) (Life Technologies) and ganciclovir $(2 \mu \mathrm{M})$ (Sigma) for 7 days. A total of 200 drug-resistant colonies were individually picked, and clones were screened for homologous integration by PCR with primers: sense (5'-TCT ATG GCT TCT GAG GCG GAA AGA A-3') and antisense (5'-CCT GTT TTG TTT CCC AGA ATG CAA GAC ATA- $3^{\prime}$ ) primer pair. The PCR was performed at $95^{\circ} \mathrm{C}$ for $2 \mathrm{~min}$; 35 cycles of $95^{\circ} \mathrm{C}$ for $15 \mathrm{~s}, 58^{\circ} \mathrm{C}$ for $30 \mathrm{~s}$, and $68^{\circ} \mathrm{C}$ for $2 \mathrm{~min}$; and 1 cycle of $68^{\circ} \mathrm{C}$ for $7 \mathrm{~min}$. Two correctly targeted ES cell clones were used to generate chimeric mice by microinjection into day $3.5 \mathrm{C} 57 \mathrm{BL} / 6$ blastocysts, which were subsequently transferred into pseudopregnant CD-1 females. Judging from the agouti coat, the two cell lines generated chimeric mice of 50 and $80 \%$ chimerism. Male chimeras were mated with ICR female mice and offspring with agouti coat color were tested for germ line transmission by PCR of tail DNA. Heterozygotes were intercrossed to produce EndoG ${ }^{+/+}$, EndoG ${ }^{+/-}$and $\mathrm{EndoG}^{-/-}$littermates.

\section{Southern and Northern blot analysis}

Southern blot analysis was carried out by using DNA extracted from liver after proteinase $\mathrm{K}$ digestion. DNA (20 $\mu \mathrm{g})$ was digested with $\mathrm{Xmnl}$ and Spel, separated on a $1 \%$ agarose gel, denatured, and neutralized by $0.5 \mathrm{M}$ $\mathrm{NaOH} / 1.5 \mathrm{M} \mathrm{NaCl}$ and $1 \mathrm{M}$ Tris $\cdot \mathrm{HCl}(\mathrm{pH} 8.0) / 1.5 \mathrm{M} \mathrm{NaCl}$, respectively, and transferred onto a nylon membrane (Nytran SuperCharge, Schleicher \& Schuell) in the presence of $10 \times$ SSC. An Xmnl-Spel fragment of $1.6 \mathrm{~kb}$ containing the exon 1 region was labeled with $\left[{ }^{32} \mathrm{P}\right] \mathrm{dATP}$ and used as probe for hybridization at $65^{\circ} \mathrm{C}$ for $16 \mathrm{~h}$. After hybridization, the membrane was washed in $2 \times \mathrm{SSC} / 0.1 \% \mathrm{SDS}(3 \times 5 \mathrm{~min}$, room temperature) and $0.2 \times \mathrm{SSC} / 0.1 \% \mathrm{SDS}\left(2 \times 10 \mathrm{~min}, 68^{\circ} \mathrm{C}\right)$, and the result was visualized using a Phospho-Imager system (Cyclone, Packard).

For Northern blot analysis, total RNA was extracted from mouse brain using the acid-phenol-guanidine isothiocyanate method (TRIzol, Invitrogen). Poly(A)-tailed RNA was fractionated from the total brain RNA by using an oligo(dT)-attached resin (Oligotex Resin, Qiagen). Poly(A) RNA $(5 \mu \mathrm{g})$ was applied to a formaldehyde-denatured agarose gel and transferred onto a nylon membrane. $\mathrm{A}^{32} \mathrm{P}$-labeled entire mouse EndoG cDNA was used to probe the membrane, and the result was visualized using a phospho-imager system.

Genotyping was performed by PCR using primers for the EndoG locus (5'-GTGCCACACCTCAACCAGAATGCC-3' and 5' -CCACGTGGCAGTGCC CACACAC- $\left.3^{\prime}\right)$ and for the neomycin cassette (5'-CTGCTAAAGCGCAT GCTCCAGACTGCCTTG-3' and 5'-GGACGACACCTTCTACCTGAGCA ACGTAGCGC-3').

\section{Antibody generation}

The rabbit polyclonal antiserum for EndoG was produced by immunizing rabbits with a synthetic peptide (coupled to keyhole limpet haemocyanin) corresponding to residues of amino-acids 279-294. The polyclonal antibody was purified through peptide-conjugated affinity chromatography with SulfoLink coupling gel (Pierce) as an immobilization support. The specificity of the anti-EndoG antibody was confirmed by the absence of EndoG bands in brain homogenate prepared from EndoG knockout mice.

\section{RT-PCR}

For RT-PCR, total RNA was isolated from the liver and testis of adult mice by using TriZol (Invitrogen), and cDNA was synthesized with SuperScript III Reverse Transcriptase (Invitrogen) following the manufacturer's protocol. This cDNA $(5 \mu \mathrm{l})$ was used as template for PCR with the following primer pairs: EndoG sense (5'-GAGCCGCGAGTCCTACGTGC TT-3'), antisense (5'-AGCCTCGGTCCTGGGCAGGAAA -3'); D2Wsu81e sense ( $5^{\prime}$-GCCCATCGAGAGCATCGAGCGGGCCTCGGGAT-3'), antisense 1 (5'-AAACCTCAAGGCTATCACTGCTGGCAGCAAGT-3') or antisense 2 (5'-GCCCATCGAGAGCATCGAGCGGGCCTCGGGAT-3').

\section{Subfractionation of mouse brain mitochondria by the swelling-shrinking procedure}

The mitochondria from mouse brain were subfractionated as described with modification. ${ }^{36}$ The mouse brain was washed in $300 \mathrm{mM}$ sucrose, $0.1 \mathrm{mM}$ EGTA, $10 \mathrm{mM}$ HEPES, pH 7.4 (buffer A), homogenized in $15 \mathrm{ml}$ of buffer $A$ using a motor-driven glass Potter-Elvehjem homogenizer with PTFE pestle. The cell debris and nuclei were centrifuged at $600 \times g$ for $5 \mathrm{~min}$, and the supernatant was filtered through 4 layers of cheesecloth. The mitochondrial fraction was obtained by centrifugation at $3300 \times g$ for $10 \mathrm{~min}$, and the resulting pellet was resuspended in $10 \mathrm{mM} \mathrm{KH}_{2} \mathrm{PO}_{4}, \mathrm{pH}$ 7.4 (buffer B) and centrifuged again. The mitochondrial pellet was resuspended in $3 \mathrm{ml}$ of buffer $B$ and incubated for $15 \mathrm{~min}$ at $4^{\circ} \mathrm{C}$ with gentle rocking. An equal volume of $32 \%(\mathrm{w} / \mathrm{v})$ sucrose, $30 \%$ glycerol $(\mathrm{v} / \mathrm{v})$, $10 \mathrm{mM} \mathrm{MgCl}_{2}$ in $10 \mathrm{mM}$ buffer $B$ was added to the suspension. After a $15 \mathrm{~min}$ incubation, the suspension was centrifuged for $10 \mathrm{~min}$ at $12000 \times g$, and the pellet (P1) and supernatant (S1) were separated. The pellet was resuspended in $3 \mathrm{ml}$ of buffer $B$ and incubated for $30 \mathrm{~min}$ at $4^{\circ} \mathrm{C}$ with rocking. The suspension was centrifuged at $160000 \times g$ for 30 min and the resulting supernatant (S2) and pellet (P2) were designated matrix (Mx) and inner membrane (IM), respectively. $S 1$ was centrifuged at $160000 \times g$, and the resulting pellet (P3) and supernatant (S3) were designated as outer membrane (OM) and intermembrane space (IMS), respectively. Protein concentration was determined and equal amount of protein was loaded for Western blotting analysis with the following antibodies: anti-VDAC (anti-porin 31HL, Ab-2, Calbiochem) for outer membrane; anticytochrome $c$ oxidase (COX) subunit IV (Molecular Probes) for inner membrane; anti-Hsp-60 (Stressgen) for matrix and antiCyt-C (BD Pharmingen) for the intermembrane space. For the $\mathrm{Na}_{2} \mathrm{CO}_{3}$ mitochondrial protein extraction, the inner membrane fraction was incubated with $0.1 \mathrm{M} \mathrm{Na}_{2} \mathrm{CO}_{3}$ in ice for $20 \mathrm{~min}$. Separation of the pellet from the released proteins was achieved by centrifugation at $160000 \times g$ for $20 \mathrm{~min}$.

\section{Histological analyses of major organs}

Two-month old mice were deeply anesthetized with pentobarbital and then perfused with phosphate buffer saline (PBS), pH 7.4 and then $4.0 \%$ paraformaldehyde in PBS, $\mathrm{pH}$ 7.4. Organs except for the brain were trimmed, embedded in OCT, and frozen for cryostat sectioning. Sections $(10-15 \mu \mathrm{m})$ were stained with hematoxylin-eosin. Some organs were paraffin-embedded, sectioned and stained with hematoxylin-eosin. 
Coronal sections $(40 \mu \mathrm{m})$ of the brain were obtained using a microtome and were stained with hematoxylin-eosin.

\section{Mouse embryonic fibroblasts (MEFs) isolation and culturing}

Embryos from matings were harvested at E 13.5. The heads and the internal organs were removed, and the remaining tissues were washed in cold PBS. The tissues were broken into smaller pieces by passing through a 10-ml syringe. The tissue pieces were then incubated in trypsin for $20 \mathrm{~min}$ and subsequently vortexed. The cells were then passed through a $100 \mu \mathrm{M}$ nylon cell strainer and plated in T-75 culture flasks with DMEM culture media containing $10 \%$ fetal bovine serum and antibiotics. MEFs were maintained at $37^{\circ} \mathrm{C}$ in a humidified atmosphere containing $5 \%$ carbon dioxide.

\section{MEFs treatment with cell death stimuli}

For the induction of cell death, MEFS $\left(1 \times 10^{4}\right.$ per well) were plated in 96 well plates and treated $24 \mathrm{~h}$ later with various cell death stimuli. All reagents used, unless indicated otherwise, were obtained from Sigma. Stock solutions of the drugs used: $N$-Methyl- $N$-Nitro- $N$-Nitrosoguanidine (MNNG), etoposide, cis-platinum(II) diamminedichloride (cisplatin), menadione, thapsigargin, tunicamycin, and staurosporine were prepared in dimethyl sulfoxide (DMSO), whereas lyophilized recombinant mouse TNF- $\alpha$ was reconstituted in water. The final concentration of DMSO in the cultures was $<0.1 \%$ at all drug concentrations and in controls. For MNNG and menadione treatment, MEFs were treated with various concentrations of drug for $15 \mathrm{~min}$, washed, returned to the normal growth media, and assayed for cell death after $24 \mathrm{~h}$ by staining with propidium iodide (PI) $(7 \mu \mathrm{M})$. The drugs cisplatin, TNF- $\alpha$ with cycloheximide $(10 \mu \mathrm{g} / \mathrm{ml})$, tunicamycin, etoposide and thapsigargin were left in culture medium, and cell death by PI staining was assayed after $24 \mathrm{~h}$ for cisplatin and TNF- $\alpha, 48 \mathrm{~h}$ for tunicamycin, and $72 \mathrm{~h}$ for etoposide and thapsigargin. Cells were stained with Hoechst 33342 (Molecular Probes) $(1 \mu \mathrm{g} / \mathrm{ml})$ and $\mathrm{PI}$, and then counted using the KS300 software (Zeiss). Cell death data were obtained from at least three independent experiments using two different cell lines per genotype.

\section{Immunocytochemistry of AIF}

Wild-type and EndoG null MEFs to be immunostained for AIF were plated on glass coverslips and treated with MNNG $24 \mathrm{~h}$ later. MEFs were treated with MNNG for $15 \mathrm{~min}$, washed, returned to the normal growth media, and then immunostained for AIF after $8 \mathrm{~h}$. For immunocytochemistry, MEFs were fixed for $20 \mathrm{~min}$ with $4 \%$ paraformaldehyde, and then immunostained with rabbit antiserum raised against AIF as described. ${ }^{23}$ This is followed by incubation with Cy3-conjugated goat anti-rabbit IgG (Jackson ImmunoResearch), and then nuclear counterstaining with $300 \mathrm{nM}$ 4',6-diamidino2-phenylindole (DAPI) (Molecular Probes). AIF immunostaining was visualized by confocal two-photon fluorescence microscopy. Translocation of AIF to the nucleus is indicated by overlay of AIF immunostaining with DAPI counterstaining.

\section{Intrastriatal injections of NMDA}

Intrastriatal injections were performed as described previously. ${ }^{37}$ Mice were anesthetized with pentobarbital ( $45 \mathrm{mg} / \mathrm{kg}$, i.p.). Using a stereotactic frame (Kopf), a hole was drilled above the right striatum (rostral, $0.5 \mathrm{~mm}$; lateral, $1.7 \mathrm{~mm}$; ventral, $3.5 \mathrm{~mm}$ from bregma), ${ }^{38}$ and NMDA (Sigma) $(66.7 \mathrm{mM}$ ) was injected for $10 \mathrm{~min}$ (volume of $0.3 \mu \mathrm{l})$. The needle was left in place for an additional $8 \mathrm{~min}$ after injection. After $48 \mathrm{~h}$, the mice were deeply anesthetized with pentobarbital and then perfused with PBS and then $0.4 \%$ paraformaldehyde in PBS, $\mathrm{pH} 7.4$. The brain was postfixed in the same fixative solution, and then cryoprotected in $20 \%$ glycerol-PBS for freezing and serial sectioning. Coronal sections $(40 \mu \mathrm{M})$ were stained with Nissl, and lesion volumes were analyzed using the SigmaScan Pro 5 software (SPSS Inc.).

\section{Acknowledgements}

We would like to thank Shaida Andrabi for help in the confocal imaging. This work was supported by USPHS NINDS NS039148. KKD was supported by a Society for Neuroscience Minority Fellowship Grant. TMD is the Leonard and Madlyn Abramson Professor in Neurodegenerative Diseases.

\section{References}

1. Liu X, Zou H, Slaughter C and Wang X (1997) DFF, a heterodimeric protein that functions downstream of caspase-3 to trigger DNA fragmentation during apoptosis. Cell 89: 175-184

2. Enari M, Sakahira H, Yokoyama H, Okawa K, Iwamatsu A and Nagata S (1998) A caspase-activated DNase that degrades DNA during apoptosis, and its inhibitor ICAD. Nature 391: 43-50

3. Sakahira H, Enari M and Nagata S (1998) Cleavage of CAD inhibitor in CAD activation and DNA degradation during apoptosis. Nature 391: 96-99

4. Halenbeck R, MacDonald H, Roulston A, Chen TT, Conroy L and Williams LT (1998) CPAN, a human nuclease regulated by the caspase-sensitive inhibitor DFF45. Curr. Biol. 8: 537-540

5. Zhang J, Liu X, Scherer DC, van Kaer L, Wang X and Xu M (1998) Resistance to DNA fragmentation and chromatin condensation in mice lacking the DNA fragmentation factor 45. Proc. Natl. Acad. Sci. USA 95: 12480-12485

6. Li LY, Luo X and Wang X (2001) Endonuclease $G$ is an apoptotic DNase when released from mitochondria. Nature 412: 95-99

7. van Loo G, Schotte P, van Gurp M, Demol H, Hoorelbeke B, Gevaert K, Rodriguez I, Ruiz-Carrillo A, Vandekerckhove J, Declercq W, Beyaert R and Vandenabeele P (2001) Endonuclease G: a mitochondrial protein released in apoptosis and involved in caspase-independent DNA degradation. Cell Death Differ. 8: 1136-1142

8. Dake E, Hofmann TJ, McIntire S, Hudson A and Zassenhaus HP (1988) Purification and properties of the major nuclease from mitochondria of Saccharomyces cerevisiae. J. Biol. Chem. 263: 7691-7702

9. von Tigerstrom RG (1982) Purification and characteristics of a mitochondrial endonuclease from the yeast Saccharomyces cerevisiae. Biochemistry 21: 6397-6403

10. Rosamond J (1981) Purification and properties of an endonuclease from the mitochondrion of Saccharomyces cerevisiae. Eur. J. Biochem. 120: 541-546

11. Cote J and Ruiz-Carrillo A (1993) Primers for mitochondrial DNA replication generated by endonuclease $\mathrm{G}$. Science 261: 765-769

12. Zassenhaus HP and Denniger G (1994) Analysis of the role of the NUC1 endo/ exonuclease in yeast mitochondrial DNA recombination. Curr. Genet. 25: 142 149

13. Ikeda S and Ozaki K (1997) Action of mitochondrial endonuclease G on DNA damaged by L-ascorbic acid, peplomycin, and cis-diamminedichloroplatinum (II). Biochem. Biophys. Res. Commun. 235: 291-294

14. Houmiel KL, Gerschenson M and Low RL (1991) Mitochondrial endonuclease activity in the rat varies markedly among tissues in relation to the rate of tissue metabolism. Biochim. Biophys. Acta 1079: 197-202

15. Zassenhaus HP, Hofmann TJ, Uthayashanker R, Vincent RD and Zona M (1988) Construction of a yeast mutant lacking the mitochondrial nuclease. Nucleic Acids Res. 16: 3283-3296 
16. Parrish J, Li L, Klotz K, Ledwich D, Wang X and Xue D (2001) Mitochondria endonuclease $G$ is important for apoptosis in C. elegans. Nature 412: 90-94

17. Zhang J, Dong M, Li L, Fan Y, Pathre P, Dong J, Lou D, Wells JM, OlivaresVillagomez D, Van Kaer L, Wang X and Xu M (2003) Endonuclease G is required for early embryogenesis and normal apoptosis in mice. Proc. Natl. Acad. Sci. USA 100: 15782-15787

18. Irvine RA, Adachi N, Shibata DK, Cassell GD, Yu K, Karanjawala ZE, Hsieh CL and Lieber MR (2005) Generation and characterization of endonuclease $G$ null mice. Mol. Cell. Biol. 25: 294-302

19. Ohsato $\mathrm{T}$, Ishihara N, Muta $\mathrm{T}$, Umeda S, Ikeda S, Mihara $\mathrm{K}$, Hamasaki $\mathrm{N}$ and Kang D (2002) Mammalian mitochondrial endonuclease G. Digestion of Rloops and localization in intermembrane space. Eur. J. Biochem. 269: 57655770

20. Uren RT, Dewson G, Bonzon C, Lithgow T, Newmeyer DD and Kluck RM (2004) Mitochondrial release of pro-apoptotic proteins: electrostatic interactions can hold cytochrome $c$ but not Smac/DIABLO to mitochondrial membranes. J. Biol. Chem. 280: 2266-2274

21. Arnoult D, Gaume B, Karbowski M, Sharpe JC, Cecconi F and Youle RJ (2003) Mitochondrial release of AIF and EndoG requires caspase activation downstream of Bax/Bak-mediated permeabilization. EMBO J. 22: 4385-4399

22. Widlak P, Li LY, Wang X and Garrard WT (2001) Action of recombinant human apoptotic endonuclease $G$ on naked DNA and chromatin substrates: cooperation with exonuclease and DNase I. J. Biol. Chem. 276: 48404-48409

23. Yu SW, Wang H, Poitras MF, Coombs C, Bowers WJ, Federoff HJ, Poirier GG Dawson TM and Dawson VL (2002) Mediation of poly(ADP-ribose) polymerase-1-dependent cell death by apoptosis-inducing factor. Science 297: 259-263

24. Siddik ZH (2003) Cisplatin: mode of cytotoxic action and molecular basis of resistance. Oncogene 22: 7265-7279

25. Burden DA, Kingma PS, Froelich-Ammon SJ, Bjornsti MA, Patchan MW, Thompson RB and Osheroff N (1996) Topoisomerase II. etoposide interactions direct the formation of drug-induced enzyme-DNA cleavage complexes. J. Biol. Chem. 271: 29238-29244

26. Thor H, Smith MT, Hartzell P, Bellomo G, Jewell SA and Orrenius S (1982) The metabolism of menadione (2-methyl-1,4-naphthoquinone) by isolated hepatocytes. A study of the implications of oxidative stress in intact cells. J. Biol. Chem. 257: 12419-12425

27. Chae HJ, Kim HR, Xu C, Bailly-Maitre B, Krajewska M, Krajewski S, Banares S, Cui J, Digicaylioglu M, Ke N, Kitada S, Monosov E, Thomas M, Kress CL, Babendure JR, Tsien RY, Lipton SA and Reed JC (2004) BI-1 regulates an apoptosis pathway linked to endoplasmic reticulum stress. Mol. Cell 15: 355366

28. Gescher A (2000) Staurosporine analogues - pharmacological toys or useful antitumour agents? Crit. Rev. Oncol. Hematol. 34: 127-135

29. Aggarwal BB (2003) Signalling pathways of the TNF superfamily: a doubleedged sword. Nat. Rev. Immunol. 3: 745-756

30. Ko MS, Threat TA, Wang X, Horton JH, Cui Y, Pryor E, Paris J, Wells-Smith J, Kitchen JR, Rowe LB, Eppig J, Satoh T, Brant L, Fujiwara H, Yotsumoto S and Nakashima $\mathrm{H}$ (1998) Genome-wide mapping of unselected transcripts from extraembryonic tissue of 7.5-day mouse embryos reveals enrichment in the tcomplex and under-representation on the X chromosome. Hum. Mol. Genet. 7: 1967-1978

31. Ko MS, Kitchen JR, Wang X, Threat TA, Hasegawa A, Sun T, Grahovac MJ, Kargul GJ, Lim MK, Cui Y, Sano Y, Tanaka T, Liang Y, Mason S, Paonessa PD, Sauls AD, DePalma GE, Sharara R, Rowe LB, Eppig J, Morrell C and Doi H (2000) Large-scale cDNA analysis reveals phased gene expression patterns during preimplantation mouse development. Development 127: 1737-1749

32. Davies AM, Hershman S, Stabley GJ, Hoek JB, Peterson J and Cahill A (2003) A Ca2+-induced mitochondrial permeability transition causes complete release of rat liver endonuclease $G$ activity from its exclusive location within the mitochondrial intermembrane space. Identification of a novel endoexonuclease activity residing within the mitochondrial matrix. Nucleic Acids Res. 31: 1364-1373

33. Parrish JZ and Xue D (2003) Functional genomic analysis of apoptotic DNA degradation in C. elegans. Mol. Cell 11: 987-996

34. Wang X, Yang C, Chai J, Shi Y and Xue D (2002) Mechanisms of AlF-mediated apoptotic DNA degradation in Caenorhabditis elegans. Science 298: 15871592

35. Krieser RJ, MacLea KS, Longnecker DS, Fields JL, Fiering S and Eastman A (2002) Deoxyribonuclease llalpha is required during the phagocytic phase of apoptosis and its loss causes perinatal lethality. Cell Death Differ. 9: 956-962

36. Hovius R, Lambrechts H, Nicolay K and de Kruijff B (1990) Improved methods to isolate and subfractionate rat liver mitochondria. Lipid composition of the inner and outer membrane. Biochim. Biophys. Acta 1021: 217-226

37. Mandir AS, Poitras MF, Berliner AR, Herring WJ, Guastella DB, Feldman A, Poirier GG, Wang ZQ, Dawson TM and Dawson VL (2000) NMDA but not nonNMDA excitotoxicity is mediated by Poly(ADP-ribose) polymerase. J. Neurosci. 20: 8005-8011

38. Franklin KBJPG (1997) Mouse Brain in Stereotaxic Coordinates (San Diego: Academic Press) 